\title{
Níveis críticos e faixas de suficiência de nutrientes derivados de métodos de avaliação do estado nutricional da palma-de-óleo
}

\author{
Gilson Sergio Bastos de Matos ${ }^{(1)}$, Antonio Rodrigues Fernandes ${ }^{(2)}$ e Paulo Guilherme Salvador Wadt(3)
}

\begin{abstract}
(1)Universidade Federal Rural da Amazônia (Ufra), Campus de Tomé-Açu, Rodovia PA-140, Km 10, Açaizal, CEP 68680-000 Tomé-Açu, PA, Brasil. E-mail: gilsonsbm@yahoo.com.br (2)Ufra, Campus Belém, Instituto de Ciências Agrárias, Avenida Presidente Tancredo Neves, № 2.501, Caixa Postal 917, Montese, CEP 66077-530 Belém, PA, Brasil. E-mail antonio.fernandes@ufra.edu.br ${ }^{(3)}$ Embrapa Rondônia, Rodovia BR-364, Km 5,5, s/no , Zona Rural, CEP 76815-800 Porto Velho, RO, Brasil. E-mail: wadt@dris.com.br.
\end{abstract}

Resumo - O objetivo deste trabalho foi determinar níveis críticos e faixas de suficiência de nutrientes para a palma-de-óleo (Elaeis spp.), a partir de métodos de avaliação do estado nutricional das plantas. Utilizaram-se os métodos chance matemática (ChM), sistema integrado de diagnose e recomendação (DRIS) e diagnose da composição nutricional (CND), além da avaliação do nível crítico obtido pela distribuição normal reduzida (DNR) em dados de produtividade e do teor de nutrientes de 144 amostras foliares de plantios no Pará. As regressões dos índices DRIS e CND com os teores foliares foram todas significativas, e o menor coeficiente de determinação foi encontrado para N. Os intervalos das faixas de suficiência obtidos com os métodos ChM, DRIS e CND nos talhões nutricionalmente equilibrados foram, em geral, mais estreitos do que os reportados na literatura, com poucos diagnósticos concordantes, sobretudo para K. Os níveis críticos obtidos pela DNR situaram-se dentro do intervalo das faixas nutricionais obtidas com os outros métodos. Os métodos ChM, DRIS, CND e DNR são adequados para a obtenção de valores de referência nutricionais e indicaram $\mathrm{N}, \mathrm{Ca}$, $\mathrm{S}, \mathrm{Mn}$ e $\mathrm{Zn}$ como os nutrientes com maior frequência de deficiência nos talhões de palma-de-óleo avaliados.

Termos para indexação: Elaeis guineensis, Elaeis oleifera, chance matemática, diagnose da composição nutricional, DRIS, nutrição de plantas.

\section{Critical levels and nutrient sufficiency ranges derived from methods for assessing the nutritional status of oil palm}

\begin{abstract}
The objective of this work was to determine critical levels and nutrient sufficiency ranges for oil palm (Elaeis spp.), using methods for assessing the nutritional status of the plants. The methods of mathematical chance (ChM), diagnosis and recommendation integrated system (DRIS), and compositional nutrient diagnosis (CND), besides the critical level obtained by the reduced normal distribution (DNR), were used in productivity data and in nutrient content of 144 leaf samples of oil palm plantations in the state of Pará, Brazil. The regressions of the DRIS and CND indexes with leaf nutrient contents were all significant, and the lowest coefficient of determination was found for $\mathrm{N}$. The sufficiency range intervals obtained with the ChM, DRIS, and CND methods in the nutritionally balanced stands were, in general, narrower than the ones reported in the literature, with few concordant diagnostics, mainly for K. The critical levels obtained with DNR were within the nutritional range intervals obtained with the other methods. ChM, DRIS, CND, and DNR are suited methods for obtaining nutritional reference values, and they indicated $\mathrm{N}, \mathrm{Ca}, \mathrm{S}, \mathrm{Mn}$, and $\mathrm{Zn}$ as the nutrients with the highest frequency of deficiency in the evaluated oil palm orchards.
\end{abstract}

Index terms: Elaeis guineensis, Elaeis oleifera, mathematical chance, compositional nutrient diagnosis, DRIS, plant nutrition.

\section{Introdução}

A produtividade da palma-de-óleo-africana (Elaeis guineensis Jacq.) nas fazendas mais tecnicizadas atinge mais de 30 toneladas por hectare de cachos de frutos frescos (CFF) (PalmElit, 2014). No Brasil, o Pará responde por mais de $90 \%$ dos plantios de palma-de-óleo, concentrados principalmente no nordeste do estado (Alves et al., 2013).
A elevada produtividade da palma-de-óleo implica alta taxa de exportação de nutrientes. Apesar disso, o conhecimento das exigências nutricionais da espécie, no Brasil, restringe-se a valores de referência obtidos por Chepote et al. (1988), na Bahia, Rodrigues (1993), no Amazonas, e Viégas \& Müller (2000), no Pará. Esses dados foram obtidos a partir de ensaios de adubação e de levantamentos nutricionais de 
longa duração que, normalmente, são dispendiosos quanto a tempo, recursos humanos e custos. Uma importante limitação desses valores de referência está relacionada ao fato de que as cultivares estudadas por aqueles autores não são mais comercializadas, e as doses de fertilizantes atualmente utilizadas foram alteradas de acordo com pesquisas conduzidas em outros países, como a Costa Rica, Malásia e Indonésia (Fairhurst \& Härdter, 2003). Esses fatores apontam para a necessidade de revalidação das recomendações oficiais de fertilização para a cultura e de verificação de sua adequabilidade às condições edafoclimáticas, nos cultivos conduzidos no Estado do Pará.

Métodos alternativos para a definição dos valores de referência baseiam-se no balanço de nutrientes e têm, como exemplos mais conhecidos, o sistema integrado de diagnose e recomendação (DRIS) (Beaufils, 1973) e o método da diagnose da composição nutricional (CND) (Parent, 2011). Outras possibilidades são os métodos da chance matemática (ChM) (Wadt et al., 2013) e da distribuição normal reduzida (DNR) (Traspadini et al., 2014).

A definição dos valores de referência, com os métodos baseados no balanço nutricional, consiste da determinação de faixas de teores nutricionais de um conjunto de plantas que sejam consideradas nutricionalmente equilibradas (Dias et al., 2013a; Kurihara et al., 2013). Para os demais métodos citados, esses valores de referência são obtidos a partir do cálculo da probabilidade de se obterem produtividades satisfatórias, em diversas classes de teores nutricionais (ChM), ou do valor da distribuição normal dos teores do nutriente que correspondem a $90 \%$ da produtividade esperada (DNR).

Esses procedimentos têm sido aplicados às culturas do arroz irrigado (Wadt et al., 2013), do algodoeiro (Serra et al., 2010), da soja (Urano et al., 2007), da laranja-pera (Camacho et al., 2012) e da cana-de-açúcar (Santos et al., 2013), entre outras. A esse respeito, para a palma-de-óleo, também já foram obtidos valores nutricionais de referência pelo DRIS, em plantios irrigados na Ásia (Behera et al., 2016), mas não há pesquisas correlatas nos cultivos brasileiros.

O objetivo deste trabalho foi determinar níveis críticos e faixas de suficiência de nutrientes para a palma-de-óleo, a partir de métodos de avaliação do estado nutricional das plantas.

\section{Material e Métodos}

Amostras foliares de palma-de-óleo foram coletadas de plantios comerciais em fase de produção, com área média de talhões de 20 ha. Foram amostrados 21 talhões no Município de Moju (02 $02^{\prime} 15^{\prime \prime S}, 48^{\circ} 34^{\prime} 56^{\prime \prime W}$ e $\left.01^{\circ} 56^{\prime} 49^{\prime \prime} \mathrm{S}, 48^{\circ} 31^{\prime} 35^{\prime \prime} \mathrm{W}\right)$ e 12 no Município de Tomé-Açu $\left(02^{\circ} 32^{\prime} 21^{\prime \prime S}\right.$, $48^{\circ} 24^{\prime} 45^{\prime \prime} \mathrm{W}$ e $02^{\circ} 32^{\prime} 21^{\prime \prime} \mathrm{S}, 48^{\circ} 24^{\prime} 45^{\prime \prime} \mathrm{W}$ ), no nordeste do Estado do Pará. A altitude média nos dois locais é de 12,5 m. O município de Moju apresenta clima do tipo Afi, conforme classificação de Köppen-Geiger, com médias anuais de temperatura e precipitação pluvial de $26^{\circ} \mathrm{C}$ e $2.500 \mathrm{~mm}$, respectivamente. ToméAçu apresenta clima do tipo Ami, de acordo com classificação de Köppen-Geiger, com médias anuais de temperatura e precipitação pluvial de $26^{\circ} \mathrm{C}$ e $2.300 \mathrm{~mm}$, respectivamente. Ambos os munícipios apresentam período menos chuvoso entre os meses de julho/agosto e dezembro.

As plantas, com idades de plantio entre 5 e 16 anos, apresentavam boa sanidade, sem registro da ocorrência de amarelecimento fatal. Os plantios foram feitos tanto com Elaeis guineensis Jacq., tipo Tenera, como com o híbrido interespecífico E. oleifera $\mathrm{x}$ E. guineensis.

A amostragem foliar foi realizada sucessivamente, entre os anos de 2010 e 2014, sempre no período de menor pluviosidade, entre agosto e novembro, tendose coletado um total de 144 amostras compostas, formadas por folhas de 25 plantas cada (amostras simples). Durante essa série de amostragens, não houve grande variação do clima, sobretudo quanto à distribuição das chuvas. As plantas amostradas situavam-se em duas linhas paralelas, a cerca de $1 / 4$ e $3 / 4$ do comprimento leste-oeste do talhão, tendo-se coletado os oito folíolos centrais - quatro de cada lado - da 17. ${ }^{\text {a }}$ folha, na contagem do ápice para a base, conforme Rodrigues et al. (2006).

Após coleta em campo, as amostras foram secas em estufa, a $65^{\circ} \mathrm{C}$, trituradas em moinho e passadas em peneira (20 mesh); em seguida, foram analisadas quanto aos teores totais de $\mathrm{N}, \mathrm{P}, \mathrm{K}, \mathrm{Ca}, \mathrm{Mg}, \mathrm{S}, \mathrm{B}, \mathrm{Cu}$, $\mathrm{Fe}, \mathrm{Mn}$ e Zn, de acordo com Malavolta et al. (1997). A produtividade acumulada $\left(\mathrm{Mg} \mathrm{ha}{ }^{1}\right)$ de cachos de frutos frescos por ano foi anotada para cada talhão e ano de amostragem.

A partir desse conjunto de dados, duas populações de referência (uma para cada faixa de idade) foram obtidas, conforme o nível de produtividade considerado 
como satisfatório, para definição das normas DRIS e CND. Os talhões foram separados em plantios jovens ( $\leq 6$ anos de plantio) e adultos ( $>6$ anos de plantio), uma vez que o potencial produtivo da palma aumenta significativamente com a idade (PalmElit, 2014). Em cada ano, a população de referência foi formada por talhões com produtividade (acumuladas) de cachos de fruto frescos (CFF) de 9 a $24 \mathrm{Mg} \mathrm{ha}^{-1}$, para as plantas jovens, e de 19 a $34 \mathrm{Mg} \mathrm{ha}^{-1}$, para as adultas. No caso da população com plantas jovens, considerou-se a média da produtividade dos talhões, bem como a produtividade média observada em plantios bem manejados na região, para a determinação da produtividade que serviria de limite para a definição das populações de alta (população de referência) e baixa produtividade. Para as plantas adultas, a definição do valor de corte seguiu o critério utilizado por Behera et al. (2016), para o DRIS, que consideraram satisfatórios talhões com palmas-de-óleo irrigadas que atingiram produtividade acima de 19 toneladas de CFF.

As normas DRIS consistiram da média e do coeficiente de variação das relações nutricionais bivariadas com maior razão de variância (Farnezi et al., 2009), enquanto as normas CND, das relações multivariadas. Nesses dois métodos, considerou-se apenas o conjunto de talhões de produção satisfatória (Parent, 2011; Teixeira et al., 2015). Os índices DRIS foram calculados com a fórmula de Beaufils (1973), e os índices CND foram calculados pelo método da relação multivariada log centrada (Parent, 2011).

Os valores de referência (deficiente, adequado e excessivo) pelo método da chance matemática (ChM) foram obtidos conforme Wadt et al. (2013), em que o número de classes foi fixado para oito, nos plantios jovens, e nove, para os plantios adultos. Nesses cálculos, também se incluiu o cálculo da chance matemática relativa $\left(\mathrm{ChMR}_{(\mathrm{i})}\right)$ e da Chance matemática média móvel $\left(\mathrm{ChMM}_{(\mathrm{i})}\right)$, em que se utilizaram as classes de teores foliares de ordem 2 a 7 (plantas jovens) e de 2 a 8 (plantas adultas).

A chance matemática relativa em cada classe "i" foi determinada conforme a expressão $\mathrm{ChMR}_{(\mathrm{i})}=$

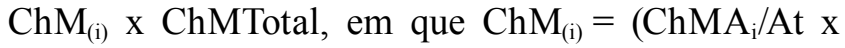
$\left.\mathrm{ChMA}_{\mathrm{i}} / \mathrm{B}_{\mathrm{i}}\right)^{0,5}, \mathrm{ChMAi} / \mathrm{At}=\mathrm{Pi} \times \mathrm{Ai} / \mathrm{At}, \mathrm{ChMAi} / \mathrm{Bi}=$ $\mathrm{Pi} \times \mathrm{Ai} / \mathrm{Ci}$ e ChMTotal $=\left[\left(\mathrm{Pm}^{2}\right) \times(\mathrm{At} / \mathrm{Ct})\right]^{0,5}$; com a seguinte descrição das variáveis: $\mathrm{ChM}_{(\mathrm{i})}$ é a chance matemática na classe "i”; ChMTotal é a chance matemática total; $\mathrm{A}_{\mathrm{i}}$ é a quantidade de lavouras de alta produtividade na classe "i"; At é o número total de lavouras de alta produtividade; $\mathrm{B}_{\mathrm{i}}$ é a produtividade média dos talhões de alta produtividade na classe "i”; Ci é o total de lavouras em cada classe "i"; Ct é o total de lavouras; Pi é a produtividade média das lavouras de alta produtividade na respectiva classe " $i$ " $\left(\mathrm{Pi}, \mathrm{kg} \mathrm{ha}^{-1}\right) ; \mathrm{Pm}$ é a produtividade média das lavouras de alta produtividade dentro de cada classe "i"; e i representa cada uma das classes de teores nutricionais que variaram de 1 a 9 .

A chance matemática média móvel de cada classe "i", exceto para a primeira e última classe de teores foliares, foi obtida pela expressão $\mathrm{ChMM}_{(\mathrm{i})}=$ (ChMR(i-1) + ChMR(i) + ChMR(i+1), em que: (i-1) corresponde ao valor de ChMR da classe de teores nutricionais anterior à classe para a qual se deseja calcular a ChMM; e $(\mathrm{i}+1)$ corresponde ao valor da ChMR da classe de teor nutricional posterior à classe para a qual se deseja calcular a ChMM.

No caso do método da chance matemática, as faixas de suficiência foram definidas conforme o critério $\mathrm{ChMM}_{50 \%}$, que consistiu da faixa de teores formada pelo limite inferior da primeira classe "i" em que a ChMM(i) apresentasse valor igual ou superior a $50 \%$ e pelo limite superior da última classe "i" em que a $\mathrm{ChMM}_{(\mathrm{i})}$ apresentasse valor igual ou superior a $50 \%$.

A faixa nutricional que indicava deficiência foi formada por teores foliares abaixo da faixa adequada no $\mathrm{ChMM}_{(\mathrm{i})}$, e a faixa de excesso, por teores foliares acima da faixa adequada.

No método da DNR, a definição dos valores de nível crítico foi feita conforme Traspadini et al. (2014). Os dados de produtividade e do quociente produtividade/ teor nutricional, quando não apresentaram distribuição normal pelo teste de Lilliefors, passaram por transformação logarítmica.

Para os métodos DRIS e CND, ajustaram-se as regressões entre os índices nutricionais e os respectivos teores foliares dos nutrientes na população de referência, conforme proposto por Wadt et al. (1998), em que os teores nutricionais foram as variáveis independentes, e seus respectivos índices foram as variáveis dependentes. Adotou-se o modelo com maior coeficiente de determinação $\left(\mathrm{R}^{2}\right)$, para a definição do teor ótimo do nutriente e, a partir disto, os limites inferior e superior da faixa de suficiência foram definidos como os teores dos nutrientes estimados quando os índices corresponderam ao 
valor nulo $\pm 2 / 3$ do desvio-padrão (Kurihara et al., 2013). Esse procedimento, portanto, consiste de um aperfeiçoamento do método originalmente proposto por Wadt et al. (1998).

As faixas de referência derivadas do DRIS, CND e ChM, bem como o nível crítico resultante do método da DNR, foram confrontadas com os valores presentes na literatura: palmas de até sete anos no médio Amazonas (Rodrigues, 1993); palmas com as faixas de idade semelhantes às obtidas no presente trabalho (Uexküll \& Fairhurst, 1991); e níveis críticos e faixas nutricionais citados por Viégas \& Müller (2000) e Malavolta et al. (1997), que foram publicados com base na compilação de valores encontrados pelos próprios autores, ou em pesquisas correlatas. Toda a literatura utilizada para fins de comparação apresentou os valores de referência para a folha n. $^{\circ} 17$, a partir da fase produtiva da palmade-óleo, que se inicia em torno dos 3,5 anos.

$\mathrm{O}$ número de nutrientes em cada classe de estado nutricional (deficiente, adequado ou excessivo) foi contabilizado e, em seguida, determinou-se o percentual de concordância dos diagnósticos do estado nutricional entre os métodos avaliados e os dados da literatura.

\section{Resultados e Discussão}

Os modelos estatísticos que representaram o ajuste entre os índices DRIS e CND e os teores de cada nutriente foram todos significativos $(p<0,01)$, nas duas faixas etárias da palma-de-óleo (Tabela 1). É importante destacar que esse procedimento não está relacionado ao uso de regressões entre a produtividade e o índice de balanço nutricional (IBN) - que é a somatória em módulo dos índices DRIS ou CND de todos os nutrientes de uma amostra -, pois, neste caso, o modelo seria falho, dado que não se deve esperar a existência de correlação entre valores de IBN e de produtividade (Wadt \& Dias, 2014).

$\mathrm{O} \mathrm{N}$ foi o nutriente com menor coeficiente de determinação entre os valores dos índices e os teores foliares $\left(\mathrm{R}^{2}\right.$ em torno de 0,60$)$, nos modelos para os dois métodos (DRIS e CND) e as duas faixas de idades testadas. Os maiores valores de $\mathrm{R}^{2}(\geq 0,95)$ foram obtidos para $\mathrm{S}$ e $\mathrm{Zn}$, em palmas jovens, e para $\mathrm{Cu}$, no caso do CND, nas palmas adultas. Esses resultados revelam elevados coeficientes de determinação, em comparação a relatos na literatura de trabalhos que utilizaram o mesmo critério, para o estabelecimento de valores de referência nutricionais, como nas culturas da videira (Teixeira et al., 2015), cana-de-açúcar (Santos et al., 2013) e laranjeira (Camacho et al., 2012; Hernandes et al., 2014), cujos valores de $\mathrm{R}^{2}$ foram superiores a $0,21,0,53$, e 0,72 , respectivamente.

Serra et al. (2010) também relataram menores coeficientes de determinação para o $\mathrm{N}$ na cultura do algodoeiro. Esses baixos valores de $\mathrm{R}^{2}$ para o $\mathrm{N}$ pode indicar que o balanço do nutriente na planta seja muito sensível às concentrações dos outros nutrientes, enquanto o balanço dos demais elementos seriam influenciados principalmente por variações em suas próprias disponibilidades, conforme sugerido para a cultura da maçã (Nachtigall \& Dechen, 2007).

Os maiores valores de $\mathrm{R}^{2}$ obtidos para plantas jovens, em comparação às adultas, sobretudo para $\mathrm{K}$, $\mathrm{Mg}, \mathrm{B}$ e Mn, evidenciam, ainda que preliminarmente, a diminuição da sensibilidade dos índices DRIS à variação dos teores dos nutrientes com $\mathrm{o}$ envelhecimento da planta, o que pode ser um indício de que processos de realocação interna tornam-se mais determinantes para o balanço nutricional do que a disponibilidade dos nutrientes para as plantas. Essa possibilidade está de acordo com a expectativa de que, em cultivos agrícolas, o teor dos nutrientes diminua com o desenvolvimento ontogenético das plantas (Áom o, 2008).

As faixas de referência de $\mathrm{N}, \mathrm{P}, \mathrm{Ca}$ e $\mathrm{Cu}$, derivadas da ChM, DRIS e CND para as plantas jovens, foram próximas entre si e semelhantes às relatadas na literatura, enquanto para os demais nutrientes houve diferenças expressivas (Tabela 2). Ressalta-se a menor amplitude da faixa de suficiência de $\mathrm{K}$, bem como os limites inferior e superior para o nutriente menores do que os relatados na literatura, principalmente na comparação com Uexküll \& Fairhurst (1991). Não se encontraram valores de faixas nutricionais para $\mathrm{Mn}$ na literatura, apenas níveis críticos, cujos valores encontram-se abaixo do limite inferior dos intervalos observados no presente estudo.

Com exceção do $\mathrm{S}$, os níveis críticos obtidos com o método DNR, em palmas jovens, estiveram próximos dos limites inferiores das faixas de referência e próximos dos níveis críticos indicados nas recomendações oficiais. Para $\mathrm{Ca}, \mathrm{B}, \mathrm{Fe}$ e, de forma mais geral, para o $\mathrm{Mn}$, esses valores foram superiores aos níveis críticos citados por Malavolta et al. (1997) e Viégas \& Müller (2000). 
Tabela 1. Modelos estatísticos usados para ajuste entre os teores foliares dos nutrientes com os índices DRIS e CND, em plantas de palma-de-óleo (Elaeis spp.) jovens e adultas ${ }^{(1)}$, em talhões comerciais no Estado do Pará.

\begin{tabular}{|c|c|c|c|c|}
\hline \multirow[t]{2}{*}{ Método } & \multicolumn{2}{|l|}{ Plantas jovens } & \multicolumn{2}{|l|}{ Plantas adultas } \\
\hline & Equação & $\mathrm{R}^{2}$ & Equação & $\mathrm{R}^{2}$ \\
\hline \multicolumn{5}{|c|}{ Nitrogênio } \\
\hline DRIS & $\mathrm{N}=28,092+0,7415^{* *} \mathrm{I}_{\mathrm{N}}$ & 0,61 & $\mathrm{~N}=28,01+0,623 * * \mathrm{I}_{\mathrm{N}}$ & 0,60 \\
\hline CND & $\mathrm{N}=27,933+1,693 * * \mathrm{I}_{\mathrm{N}}$ & 0,62 & $\mathrm{~N}=28,107+1,8124 * * \mathrm{I}_{\mathrm{N}}$ & 0,60 \\
\hline \multicolumn{5}{|c|}{ Fósforo } \\
\hline DRIS & $\mathrm{P}=1,804+0,501 * * \mathrm{I}_{\mathrm{P}}$ & 0,77 & $\mathrm{P}=1,7194+0,049 * * \mathrm{I}_{\mathrm{P}}$ & 0,77 \\
\hline CND & $\mathrm{P}=1,780+0,178^{* *} \mathrm{I}_{\mathrm{P}}$ & 0,81 & $\mathrm{P}=1,711+0,205 * * \mathrm{I}_{\mathrm{P}}$ & 0,80 \\
\hline \multicolumn{5}{|c|}{ Potássio } \\
\hline DRIS & $\mathrm{K}=8,074+0,291 * * \mathrm{I}_{\mathrm{K}}$ & 0,92 & $\mathrm{~K}=7,0311+0,2821 * * \mathrm{I}_{\mathrm{K}}$ & 0,86 \\
\hline CND & $\mathrm{K}=7,745+1,677 * \mathrm{I}_{\mathrm{K}}$ & 0,94 & $\mathrm{~K}=7,143+1,323 * * \mathrm{I}_{\mathrm{K}}$ & 0,85 \\
\hline \multicolumn{5}{|c|}{ Cálcio } \\
\hline DRIS & $\mathrm{Ca}=8,828+0,3448 * * \mathrm{I}_{\mathrm{Ca}}$ & 0,88 & $\mathrm{Ca}=7,88+0,297 * * \mathrm{I}_{\mathrm{Ca}}$ & 0,88 \\
\hline CND & $\mathrm{Ca}=8,985+1,367 * * \mathrm{I}_{\mathrm{Ca}}$ & 0,91 & $\mathrm{Ca}=8,101+1,323 * * \mathrm{I}_{\mathrm{Ca}}$ & 0,90 \\
\hline \multicolumn{5}{|c|}{ Magnésio } \\
\hline DRIS & $\mathrm{Mg}=2,591+0,1063 * * \mathrm{I}_{\mathrm{Mg}}$ & 0,90 & $\mathrm{Mg}=2,219+0,072 * * \mathrm{I}_{\mathrm{Mg}}$ & 0,80 \\
\hline CND & $\mathrm{Mg}=2,4867+0,4912 * * \mathrm{I}_{\mathrm{Mg}}$ & 0,92 & $\mathrm{Mg}=2,1746+0,293^{*}{ }_{\mathrm{IMg}}$ & 0,81 \\
\hline \multicolumn{5}{|c|}{ Enxofre } \\
\hline DRIS & $\mathrm{S}=1,419+0,0871 * * \mathrm{I}_{\mathrm{S}}$ & 0,96 & $\mathrm{~S}=1,331+0,078^{* *} \mathrm{I}_{\mathrm{S}}$ & 0,95 \\
\hline CND & $\mathrm{S}=1,5575+0,6187^{* *} \mathrm{I}_{\mathrm{S}}$ & 0,96 & $\mathrm{~S}=1,419+0,514^{* *} \mathrm{I}_{\mathrm{S}}$ & 0,95 \\
\hline \multicolumn{5}{|c|}{ Boro } \\
\hline DRIS & $\mathrm{B}=24,894+0,7935 * * \mathrm{I}_{\mathrm{B}}$ & 0,91 & $\mathrm{~B}=24,02+0,9 * * \mathrm{I}_{\mathrm{B}}$ & 0,88 \\
\hline CND & $\mathrm{B}=24,523+4,7247 * * \mathrm{I}_{\mathrm{B}}$ & 0,94 & $\mathrm{~B}=23,979+3,881 * * \mathrm{I}_{\mathrm{B}}$ & 0,88 \\
\hline \multicolumn{5}{|c|}{ Cobre } \\
\hline DRIS & $\mathrm{Cu}=5,470+0,2174 * * \mathrm{I}_{\mathrm{Cu}}$ & 0,94 & $\mathrm{Cu}=5,976+0,248^{* *} \mathrm{I}_{\mathrm{Cu}}$ & 0,94 \\
\hline CND & $\mathrm{Cu}=5,335+0,997 * * \mathrm{I}_{\mathrm{Cu}}$ & 0,93 & $\mathrm{Cu}=5,680+1,540 * * \mathrm{I}_{\mathrm{Cu}}$ & 0,95 \\
\hline \multicolumn{5}{|c|}{ Ferro } \\
\hline DRIS & $\mathrm{Fe}=93,986+3,8062 * * \mathrm{I}_{\mathrm{Fe}}$ & 0,93 & $\mathrm{Fe}=91,581+3,839 * * \mathrm{I}_{\mathrm{Fe}}$ & 0,91 \\
\hline $\mathrm{CND}$ & $\mathrm{Fe}=94,641+19,569 * * \mathrm{I}_{\mathrm{Fe}}$ & 0,91 & $\mathrm{Fe}=90,759+18,013 * * \mathrm{I}_{\mathrm{Fe}}$ & 0,89 \\
\hline \multicolumn{5}{|c|}{ Manganês } \\
\hline DRIS & $\mathrm{Mn}=284,9+11,006^{* *} \mathrm{I}_{\mathrm{Mn}}$ & 0,94 & $\mathrm{Mn}=256,4+8,4891 * * \mathrm{I}_{\mathrm{Mn}}$ & 0,81 \\
\hline CND & $\mathrm{Mn}=292+64,979 * * \mathrm{I}_{\mathrm{M}} \mathrm{n}$ & 0,94 & $\mathrm{Mn}=260,6+39,68 * * \mathrm{I}_{\mathrm{Mn}}$ & 0,88 \\
\hline \multicolumn{5}{|c|}{ Zinco } \\
\hline DRIS & $\mathrm{Zn}=17,032+0,8794 * * \mathrm{I}_{\mathrm{Zn}}$ & 0,95 & $\mathrm{Zn}=17,21+0,807 * * \mathrm{I}_{\mathrm{Zn}}$ & 0,91 \\
\hline CND & $\mathrm{Zn}=17,946+5,432 * * \mathrm{I}_{\mathrm{Mn}}$ & 0,96 & $\mathrm{Zn}=16,67+3,799 * * \mathrm{I}_{\mathrm{Zn}}$ & 0,93 \\
\hline
\end{tabular}

(1)Plantas jovens, com menos de seis anos após o plantio; plantas adultas, mais de seis anos após o plantio. **Significativo a 1\% de probabilidade. 
Nas plantas adultas, as faixas de suficiência obtidas foram semelhantes às relatadas na literatura, para $\mathrm{P}, \mathrm{Ca}, \mathrm{S}$ e $\mathrm{Cu}$. Para $\mathrm{N}$ e $\mathrm{K}$, os intervalos foram mais estreitos e, para os demais elementos, tanto as faixas, obtidas com ChM, DRIS e CND, como os níveis críticos, obtidos com a DNR, foram semelhantes aos valores presentes na literatura para plantas jovens.

Em geral, com exceção do $\mathrm{N}$ e do $\mathrm{Cu}$, cujos limites superiores foram aumentados da fase jovem para a adulta, os valores de faixas nutricionais e nível crítico diminuíram. Esses resultados têm a mesma explicação que a dada para a diminuição dos $\mathrm{R}^{2}$ dos modelos ajustados com o aumento da idade das plantas (Tabela 1).

Faixas de suficiência com menores amplitudes e limites do que os relatados na literatura, como no caso do $\mathrm{K}$, têm sido comumente observadas em trabalhos com os métodos ChM, DRIS e CND sobre algodoeiro
(Serra et al., 2010), cana-de-açúcar (Santos et al., 2013) e algodoeiro e soja (Kurihara et al., 2013).

Para a palma-de-óleo, na Índia, as faixas de suficiência derivadas com o DRIS para $\mathrm{K}$ também foram inferiores aos antigos valores publicados, mas semelhantes aos observados no presente estudo, entre 7,8 a 9,1 $\mathrm{g} \mathrm{kg}^{-1}$ (Behera et al., 2016). São frequentes as diferenças entre as faixas nutricionais obtidas em ensaios de calibração e aquelas com os métodos de avaliação do estado nutricional, em talhões comerciais. Entretanto, os dados obtidos de campos comerciais geralmente consideram as condições locais, ou seja, são mais adequados para serem usados nas regiões onde foram obtidos do que os valores determinados em condições controladas, geralmente em condições edafoclimáticas e agronômicas diferentes (Urano et al., 2007). Entretanto, Camacho et al. (2012) alertaram que a obtenção de valores de referência nutricionais

Tabela 2. Faixas de suficiência para os teores foliares de nutrientes, em plantas de palma-de-óleo jovens e adultas ${ }^{(1)}$, geradas a partir do sistema integrado de diagnose e recomendação (DRIS), da composição da diagnose nutricional (CND) e da chance matemática $(\mathrm{ChM})$, bem como valores de nível crítico derivados a partir do método da distribuição normal reduzida (DNR), em talhões comerciais no nordeste do Estado do Pará, além das faixas suficiência presentes na literatura.

\begin{tabular}{|c|c|c|c|c|c|c|c|c|c|c|}
\hline $\mathrm{N}$ & $\mathrm{P}$ & $\mathrm{K}$ & $\mathrm{Ca}$ & $\mathrm{Mg}$ & $\mathrm{S}$ & $\mathrm{B}$ & $\mathrm{Cu}$ & $\mathrm{Fe}$ & $\mathrm{Mn}$ & $\mathrm{Zn}$ \\
\hline & & ----- $(\mathrm{g} \mathrm{kg}$ & ------------ & ----------- & ----- & ---------- & --------- & $--\left(\mathrm{mg} \mathrm{kg}^{-1}\right)$ & ----------- & ------------ \\
\hline \multicolumn{11}{|c|}{ ChM, plantas jovens } \\
\hline $28,3-30,7$ & $1,5-2,9$ & $5,0-7,1$ & $8,6-11,4$ & $2,-2,5$ & $1,3-2,7$ & $19,4-31,8$ & $4,1-6,5$ & $76-106$ & $232-296$ & $14,5-28,1$ \\
\hline \multicolumn{11}{|c|}{ DRIS, plantas jovens } \\
\hline $25,6-30,7$ & $1,6-2,0$ & $7,1-9$ & $7,6-10$ & $2,2-2,9$ & $1,1-1,7$ & $22,5-27,5$ & $4,7-6,2$ & $81-107$ & $248-322$ & $14,1-20$ \\
\hline \multicolumn{11}{|c|}{$\mathrm{CND}$, plantas jovens } \\
\hline $26,8-29,1$ & $1,6-1,9$ & $6,6-8,8$ & $8,0-9,8$ & $2,1-2,8$ & $1,1-1,9$ & $21,3-27,6$ & $4,6-6,0$ & $82-108$ & 249-335 & $14,6-21,3$ \\
\hline \multicolumn{11}{|c|}{ DNR, plantas jovens } \\
\hline 27,5 & 1,7 & 7,5 & 8,6 & 2,4 & 1,2 & 24,3 & 5,2 & 86,9 & 258,1 & 15,4 \\
\hline \multicolumn{11}{|c|}{ ChM, plantas adultas } \\
\hline $27,7-31,6$ & $1,3-2,0$ & $5,9-7,4$ & $7,1-9,4$ & $1,9-2,5$ & $1-2,7$ & $17,7-29,6$ & $3,5-5,6$ & $107-115$ & $177-349$ & $11,1-21,2$ \\
\hline \multicolumn{11}{|c|}{ DRIS, plantas adultas } \\
\hline $25,9-30,1$ & $1,6-1,9$ & $6,1-8,9$ & $6,9-8,9$ & $1,9-2,5$ & $1,1-1,6$ & $21-27$ & $5,1-6,8$ & $79-104$ & $228-285$ & $14,5-19,9$ \\
\hline \multicolumn{11}{|c|}{ ChM, plantas adultas } \\
\hline $26,9-29,3$ & $1,6-1,8$ & $6,2-8,0$ & $7,1-9,0$ & $1,9-2,3$ & $1,1-1,8$ & $21,4-26,6$ & $4,7-6,6$ & $79-103$ & $234-288$ & $14,05-19,3$ \\
\hline \multicolumn{11}{|c|}{ DNR, plantas adultas } \\
\hline 27,8 & 1,7 & 7,0 & 7,4 & 2,1 & 1,2 & 22,7 & 5,0 & 85,8 & 243,6 & 15,5 \\
\hline \multicolumn{11}{|c|}{ Uexküll \& Fairhurst (1991), plantas jovens } \\
\hline $26-29$ & $1,6-1,9$ & 11-13, & $5,0-7,0$ & $3,0-4,5$ & $2,5-4$ & $15-25$ & $5,0-8,0$ & - & - & $12-18$ \\
\hline \multicolumn{11}{|c|}{ Uexküll \& Fairhurst (1991), plantas adultas } \\
\hline $24-28$ & $1,5-1,8$ & $9,0-12$, & $5,0-7,5$ & $2,5-4,0$ & $2,5-4$ & $15-25$ & $5,0-8,0$ & - & - & $12-18$ \\
\hline \multicolumn{11}{|c|}{ Rodrigues (1993) } \\
\hline $22,2-27,0$ & $1,31-1,76$ & $5,25-13,46$ & $7,28-10,8$ & $2,00-3,69$ & $1,7-2,1$ & $15,7-26,7$ & $3,4-7,0$ & - & - & $8,4-12,9$ \\
\hline \multicolumn{11}{|c|}{ Malavolta et al. (1997) } \\
\hline 25 & 1,5 & 10,0 & 6,0 & 2,4 & 2,1 & $12,0-14,0$ & 10,0 & $50-250$ & 50,0 & 18,0 \\
\hline \multicolumn{11}{|c|}{ Viégas \& Muller (2000) } \\
\hline 25 & 1,5 & 10,0 & 2,4 & 6 & 2,2 & 12 & - & 50 & - & - \\
\hline
\end{tabular}

${ }^{(1)}$ Plantas jovens, com menos de seis anos após o plantio; plantas adultas, mais de seis anos após o plantio. 
precisos, a partir de dados de campo, depende de uma ampla variação dos teores de cada nutriente, o que não foi observado no presente trabalho para o K.

As coletas foliares, realizadas em período próximo do pico da safra, que no Estado do Pará ocorre entre os meses de outubro e novembro, podem ter influenciado os teores foliares de $\mathrm{K}$, que teriam sido mais baixos, pelo fato de esse nutriente ser o mais exportado nos cachos (Viégas \& Müller, 2000). Portanto, a fim de validar os resultados apresentados, ou aumentar a precisão dos métodos ChM, DRIS e CND, é importante que outras avaliações sejam realizadas, para aumentar o banco de dados e testar o efeito de amostragens em várias épocas do ano. Nesse sentido, tanto o DRIS, na cultura do maracujá (Carvalho et al., 2011), como o CND, na de arroz de sequeiro (Tomio et al., 2015), tiveram seus padrões nutricionais alterados, quando a amostragem foi feita em período próximo da época de colheita.

No Brasil, apesar dos trabalhos de Malavolta et al. (1997) e Viégas \& Müller (2000), que propuseram teores adequados e níveis críticos para Fe e Mn com base na literatura, as faixas de suficiência para esses micronutrientes ainda não haviam sido obtidas com dados do País. No entanto, em avaliações de campo em Tailândia, PA, que é um Município adjacente a Tomé-Açu, os valores encontrados para todos os micronutrientes situaram-se dentro ou no entorno dos apresentados na Tabela 2 (Viégas \& Müller, 2000) e, portanto, refletiram satisfatoriamente a realidade local. Nas faixas derivadas do DRIS, na Índia, observaramse intervalos que revelam limites superiores muito altos para esses micronutrientes (Behera et al., 2016): $82,8-936 \mathrm{mg} \mathrm{kg}^{-1}$, para Fe; 82,5-681 $\mathrm{mg} \mathrm{kg}^{-1}$, para Mn; e 33,6-58,6 mg kg-1, para $\mathrm{Zn}$. Desses valores, apenas foi possível verificar a intercessão com as faixas aqui obtidas para o Fe.

Independentemente da idade, as faixas de suficiência relatadas por Uexkül \& Fairhurst (1991) revelaram maior deficiência de $\mathrm{K}, \mathrm{S}, \mathrm{Mg}$ e $\mathrm{N}$, e as relatadas por Rodrigues (1993) mostraram maior deficiência quanto ao $\mathrm{S} \mathrm{e} \mathrm{Ca}$ (Tabela 3). As doses de fertilizantes, utilizadas nos experimentos de calibração pelos primeiros autores, na Ásia, foram superiores às utilizadas por Rodrigues (1993), no Estado do Amazonas. Além disso, a produtividade média dos plantios da Indonésia e Malásia esteve acima dos padrões brasileiros (Fairhurst \& Härdter, 2003), o que é indicativo de que as faixas propostas por Uexküll \& Fairhurst (1991) são mais adequadas para palmas com elevado rendimento de cachos. Os dados de referência nutricional, propostos por esses últimos autores, têm sido comumente utilizadas nos plantios comerciais no Pará. No entanto, é importante observar que os valores propostos por Rodrigues (1993) levam em consideração condições edafoclimáticas mais próximas às constatadas nesses plantios. As cultivares utilizadas em ambos os trabalhos, no entanto, já estão ultrapassadas.

O diagnóstico realizado nas plantas jovens, pelas faixas de suficiência provenientes da ChM, mostrou que os nutrientes com maior frequência de deficiência foram: $\quad \mathrm{N}>\mathrm{S}>\mathrm{Ca}>\mathrm{Zn}>\mathrm{Mn}>\mathrm{Fe}>\mathrm{B}=\mathrm{Cu}>\mathrm{P}=\mathrm{Mg}>\mathrm{K}$; para as faixas derivadas do DRIS, eles foram $\mathrm{Ca}>\mathrm{B}>\mathrm{Fe}>\mathrm{S}=\mathrm{Mn}=\mathrm{Zn}>\mathrm{N}>\mathrm{P}=\mathrm{Mg}>\mathrm{K}>\mathrm{Cu}$; e, para oCND, $\mathrm{Ca}>\mathrm{N}>\mathrm{Zn}>\mathrm{Fe}>\mathrm{S}=\mathrm{Mn}>\mathrm{B}>\mathrm{P}>\mathrm{K}>\mathrm{Mg}>\mathrm{Cu}$ (Tabela 3). No diagnóstico com plantas adultas, o método $\mathrm{ChM}$ revelou $\mathrm{Fe}>\mathrm{N}>\mathrm{Ca}>\mathrm{K}>\mathrm{S}>\mathrm{Mg}=\mathrm{Mn}>\mathrm{B}>\mathrm{Cu}>\mathrm{P}=\mathrm{Zn} ; \quad 0$ DRIS, $\quad \mathrm{N}=\mathrm{Zn}>\mathrm{Mn}>\mathrm{S}>\mathrm{P}=\mathrm{Ca}>\mathrm{B}=\mathrm{Fe}>\mathrm{Cu}>\mathrm{K}>\mathrm{Mg}$; e o $\mathrm{CND}, \mathrm{N}>\mathrm{Mn}>\mathrm{Ca}>\mathrm{S}>\mathrm{B}=\mathrm{Zn}>\mathrm{P}>\mathrm{Fe}>\mathrm{K}>\mathrm{Cu}>\mathrm{Mg}$.

$\mathrm{OCa}$ figurou entre os nutrientes mais frequentemente deficientes nos talhões de palma-de-óleo avaliados, tanto com os valores de referência gerados no presente trabalho como com os presentes em Rodrigues (1993). O $\mathrm{N}$ também aparece entre os frequentemente deficientes nas áreas avaliadas. Pôde-se observar, ainda, que os diagnósticos feitos a partir das faixas geradas com ChM, DRIS e CND ressaltaram a frequência de deficiência de micronutrientes. Em plantios na Índia, o $\mathrm{Mn}$ foi o nutriente mais deficiente, depois do $\mathrm{K}$ e do $\mathrm{N}$ (Behera et al., 2016).

De acordo com esses dados, deve-se dispensar atenção especial ao manejo nutricional com $\mathrm{N}$ e $\mathrm{Ca}$, nos plantios de palma-de-óleo do Pará. Na região, é comum a constatação visual de deficiência de $\mathrm{N}$ nas plantas, o que está relacionado ao fato de ele ser o segundo nutriente mais requerido pela cultura (Viégas \& Müller, 2000). Já a deficiência de Ca pode estar associada à ausência do nutriente, em formulações de fertilizantes comerciais mais concentradas, e ao fato de que as empresas que cultivam a palma-de-óleo na região nem sempre fazem a calagem do solo.

O percentual de concordância dos diagnósticos, realizados com as faixas geradas a partir de ChM, DRIS e CND, e com as presentes na literatura, em geral, foi baixo, principalmente os de $\mathrm{K}, \mathrm{Ca}$ e $\mathrm{Mg}$ (Tabela 4), na comparação com diagnósticos realizados a partir das faixas presentes em Uexkül \& Fairhurst (1991). Esse 
Tabela 3. Número de talhões com status nutricional deficiente (D), adequado (A) e em excesso (E), de acordo com as faixas de suficiência relatadas na literatura e com as geradas a partir dos métodos da chance matemática (ChM), do sistema integrado de diagnose e recomendação (DRIS) e da diagnose da composição nutricional (CND), em talhões comerciais de plantas jovens e adultas ${ }^{(1)}$ de palma-de-óleo (Elaeis spp.), no nordeste do Estado do Pará.

\begin{tabular}{|c|c|c|c|c|c|c|c|c|c|c|c|c|}
\hline \multicolumn{7}{|c|}{ Plantas jovens } & \multicolumn{6}{|c|}{ Plantas adultas } \\
\hline & $\mathrm{ChM}$ & DRIS & CND & $\begin{array}{c}\text { Rodrigues } \\
\text { (1993) }\end{array}$ & $\begin{array}{c}\text { Uexküll \& } \\
\text { Fairhurst (1991) }\end{array}$ & $\begin{array}{l}\text { Malavolta } \\
\text { et al. (1997) }\end{array}$ & $\mathrm{ChM}$ & DRIS & $\mathrm{CND}$ & $\begin{array}{c}\text { Rodrigues } \\
\text { (1993) }\end{array}$ & $\begin{array}{c}\text { Uexküll \& } \\
\text { Fairhurst (1991) }\end{array}$ & $\begin{array}{l}\text { Malavolta } \\
\text { et al. (1997) }\end{array}$ \\
\hline \multicolumn{13}{|c|}{ Nitrogênio } \\
\hline $\mathrm{D}$ & 43 & 20 & 30 & 0 & 24 & - & 53 & 30 & 39 & 2 & 12 & - \\
\hline A & 14 & 38 & 16 & 34 & 22 & - & 25 & 44 & 28 & 42 & 46 & - \\
\hline $\mathrm{E}$ & 3 & 3 & 15 & 27 & 15 & - & 5 & 9 & 16 & 39 & 25 & - \\
\hline \multicolumn{13}{|c|}{ Fósforo } \\
\hline $\mathrm{D}$ & 5 & 14 & 14 & 2 & 14 & - & 1 & 25 & 25 & 3 & 11 & - \\
\hline A & 55 & 40 & 33 & 28 & 33 & - & 74 & 43 & 24 & 43 & 38 & - \\
\hline $\mathrm{E}$ & 1 & 7 & 14 & 31 & 14 & - & 8 & 15 & 34 & 37 & 34 & - \\
\hline \multicolumn{13}{|c|}{ Potássio } \\
\hline $\mathrm{D}$ & 3 & 12 & 10 & 3 & 60 & - & 17 & 20 & 20 & 2 & 82 & - \\
\hline A & 9 & 35 & 34 & 58 & 1 & - & 29 & 47 & 38 & 81 & 1 & - \\
\hline $\mathrm{E}$ & 49 & 14 & 17 & 0 & 0 & - & 37 & 16 & 25 & 0 & 0 & - \\
\hline \multicolumn{13}{|c|}{ Cálcio } \\
\hline $\mathrm{D}$ & 37 & 35 & 37 & 30 & 1 & - & 33 & 25 & 33 & 37 & 2 & - \\
\hline A & 21 & 15 & 13 & 26 & 17 & - & 42 & 37 & 31 & 43 & 30 & - \\
\hline E & 3 & 11 & 11 & 5 & 42 & - & 8 & 21 & 19 & 3 & 51 & - \\
\hline \multicolumn{13}{|c|}{ Magnésio } \\
\hline D & 5 & 14 & 9 & 6 & 48 & - & 14 & 14 & 14 & 19 & 79 & - \\
\hline A & 19 & 31 & 32 & 54 & 12 & - & 48 & 48 & 30 & 63 & 4 & - \\
\hline E & 37 & 16 & 20 & 1 & 1 & - & 21 & 21 & 39 & 1 & 0 & - \\
\hline \multicolumn{13}{|c|}{ Enxofre } \\
\hline $\mathrm{D}$ & 38 & 23 & 23 & 51 & 56 & - & 15 & 27 & 27 & 70 & 77 & - \\
\hline A & 22 & 28 & 28 & 1 & 5 & - & 65 & 42 & 43 & 2 & 6 & - \\
\hline E & 1 & 10 & 10 & 9 & 0 & - & 3 & 14 & 13 & 11 & 0 & - \\
\hline \multicolumn{13}{|c|}{ Boro } \\
\hline D & 6 & 26 & 22 & 4 & 3 & 0 & 7 & 23 & 26 & 3 & 1 & 0 \\
\hline A & 49 & 19 & 23 & 39 & 31 & 2 & 63 & 36 & 31 & 55 & 48 & 1 \\
\hline E & 6 & 16 & 16 & 18 & 27 & 59 & 13 & 24 & 26 & 25 & 34 & 82 \\
\hline \multicolumn{13}{|c|}{ Cobre } \\
\hline D & 6 & 9 & 7 & 0 & 16 & - & 4 & 22 & 15 & 3 & 18 & \\
\hline A & 37 & 31 & 33 & 51 & 45 & - & 31 & 33 & 36 & 62 & 61 & \\
\hline $\mathrm{E}$ & 18 & 21 & 21 & 10 & 0 & - & 48 & 28 & 32 & 18 & 4 & \\
\hline \multicolumn{13}{|c|}{ Ferro } \\
\hline D & 18 & 24 & 24 & - & - & 2 & 61 & 23 & 23 & - & - & 0 \\
\hline A & 31 & 25 & 25 & - & - & 59 & 7 & 38 & 38 & - & - & 83 \\
\hline $\mathrm{E}$ & 12 & 12 & 12 & - & - & 0 & 15 & 22 & 22 & - & - & 0 \\
\hline \multicolumn{13}{|c|}{ Manganês } \\
\hline D & 22 & 23 & 23 & - & - & - & 14 & 29 & 35 & - & - & \\
\hline A & 11 & 23 & 24 & - & - & - & 65 & 32 & 28 & - & - & \\
\hline E & 28 & 15 & 14 & - & - & - & 4 & 22 & 20 & - & - & \\
\hline \multicolumn{13}{|c|}{ Zinco } \\
\hline $\mathrm{D}$ & 26 & 23 & 26 & 0 & 0 & - & 1 & 30 & 26 & 0 & 4 & - \\
\hline A & 31 & 26 & 27 & 2 & 45 & - & 63 & 29 & 32 & 8 & 48 & - \\
\hline $\mathrm{E}$ & 4 & 12 & 8 & 59 & 16 & - & 19 & 24 & 25 & 75 & 31 & - \\
\hline
\end{tabular}

(1)Plantas jovens, com menos de seis anos após o plantio; plantas adultas, mais de seis anos após o plantio. 
Tabela 4. Percentual de concordância entre os diagnósticos realizados, com as faixas de suficiência relatadas na literatura e as obtidas a partir dos métodos da chance matemática (ChM), do sistema integrado de diagnose e recomendação (DRIS) e da diagnose da composição nutricional (CND), em talhões comerciais de plantas jovens e adultas ${ }^{(1)}$ de palma-de-óleo (Elaeis spp.), no nordeste do Estado do Pará.

\begin{tabular}{|c|c|c|c|c|c|c|c|c|c|c|c|c|}
\hline Faixas comparadas & $\mathrm{N}$ & $\mathrm{P}$ & $\mathrm{K}$ & $\mathrm{Ca}$ & $\mathrm{Mg}$ & $\mathrm{S}$ & $\mathrm{B}$ & $\mathrm{Cu}$ & $\mathrm{Fe}$ & $\mathrm{Mn}$ & $\mathrm{Zn}$ & Média \\
\hline & \multicolumn{12}{|c|}{ Plantas jovens } \\
\hline ChM x DRIS & 61 & 75 & 33 & 84 & 51 & 61 & 51 & 90 & 90 & 54 & 82 & 66 \\
\hline ChM x CND & 61 & 64 & 87 & 87 & 25 & 61 & 57 & 93 & 90 & 77 & 93 & 72 \\
\hline ChM x Rodrigues (1993) & 5 & 46 & 85 & 85 & 23 & 66 & 77 & 77 & - & - & 7 & 52 \\
\hline ChM x Uexküll \& Fairhurst (1991) & 48 & 64 & 7 & 7 & 51 & 69 & 10 & 54 & - & - & 38 & 38 \\
\hline ChM x Malavolta et al. (1997) & - & - & - & - & - & - & 10 & - & 54 & - & - & 32 \\
\hline DRIS x CND & 62 & 87 & 90 & 95 & 84 & 98 & 92 & 95 & 98 & 97 & 89 & 90 \\
\hline DRIS x Rodrigues (1993) & 26 & 39 & 62 & 82 & 61 & 51 & 59 & 67 & - & - & 20 & 52 \\
\hline DRIS x Uexküll \& Fairhurst (1991) & 72 & 87 & 20 & 20 & 25 & 36 & 43 & 54 & - & - & 54 & 46 \\
\hline DRIS x Malavolta et al. (1997) & - & - & - & - & - & - & 26 & - & 43 & - & 13 & 27 \\
\hline CND x Rodrigues (1993) & 30 & 51 & 61 & 79 & 62 & 51 & 66 & 70 & - & - & 44 & 57 \\
\hline CND x Uexküll \& Fairhurst (1991) & 89 & 98 & 16 & 20 & 16 & 36 & 49 & 51 & - & - & - & 47 \\
\hline CND x Malavolta et al. (1997) & - & - & - & - & - & - & 26 & - & 43 & - & - & 34 \\
\hline \multirow[t]{2}{*}{ Rodrigues (1993) x Uexküll \& Fairhurst (1991) } & 39 & 51 & 28 & 10 & 31 & 82 & 82 & 56 & - & - & 30 & - \\
\hline & \multicolumn{12}{|c|}{ Plantas adultas } \\
\hline ChM x DRIS & 67 & 61 & 71 & 75 & 100 & 72 & 67 & 54 & 46 & 60 & 59 & 67 \\
\hline $\mathrm{ChM} \times \mathrm{CND}$ & 70 & 82 & 82 & 87 & 78 & 73 & 61 & 67 & 46 & 55 & 63 & 70 \\
\hline ChM x Rodrigues (1993) & 8 & 54 & 37 & 89 & 70 & 24 & 81 & 63 & - & - & 31 & 51 \\
\hline ChM x Uexküll \& Fairhurst (1991) & 27 & 2.4 & 20 & 12 & 17 & 22 & 67 & 30 & - & - & 82 & 31 \\
\hline ChM x Malavolta et al. (1997) & - & - & - & - & - & - & 16 & - & 8 & - & - & 12 \\
\hline DRIS $\times$ CND & 81 & 77 & 89 & 88 & 78 & 99 & 94 & 87 & 100 & 90 & 94 & 89 \\
\hline DRIS x Rodrigues (1993) & 30 & 47 & 59 & 64 & 70 & 46 & 75 & 65 & - & - & 29 & 54 \\
\hline DRIS x Uexküll \& Fairhurst (1991) & 59 & 60 & 24 & 36 & 17 & 33 & 61 & 66 & - & - & 60 & 46 \\
\hline DRIS x Malavolta et al. (1997) & - & - & - & - & - & - & 29 & & 46 & - & - & 37 \\
\hline CND x Rodrigues (1993) & 28 & 70 & 48 & 76 & 48 & 46 & 71 & 69 & - & - & 30 & 54 \\
\hline CND x Uexküll \& Fairhurst (1991) & 28 & 31 & 24 & 25 & 17 & 33 & 60 & 63 & - & - & 66 & 39 \\
\hline CND x Malavolta et al. (1997) & - & - & - & - & - & - & 31 & - & - & - & - & 31 \\
\hline Rodrigues (1993) x Uexküll \& Fairhurst (1991) & 71 & 87 & 4 & 6 & 27 & 84 & 87 & 65 & - & - & 42 & 52 \\
\hline
\end{tabular}

(1)Plantas jovens, com menos de seis anos após o plantio; plantas adultas, mais de seis anos após o plantio.

tipo de divergência nos diagnósticos foi relatado para a cultura da laranjeira (Dias et al., 2013b) e do cafeeiro (Farnezi et al., 2009), para faixas derivadas do DRIS, bem como em lavouras arrozeiras irrigadas, para faixas derivadas de ChM e CND (Wadt et al., 2013).

As faixas de suficiência propostas por Uexküll \& Fairhurst (1991) e por Rodrigues (1993) divergiram amplamente, com exceção das obtidas para S e B, em plantas jovens, e das obtidas para esses nutrientes mais o P, nas plantas adultas. É importante observar esses contrastes nas recomendações oficiais, pois tal problema ressalta a necessidade de obtenção de valores de referência locais.

A concordância muito baixa dos diagnósticos quanto ao K, entre as faixas geradas a partir de ChM, DRIS e CND e as propostas por Uexkül \& Fairhurst (1991), pode ser atribuída às estreitas faixas obtidas com os métodos de monitoramento nutricional (Tabela 2), indício de que os valores obtidos no presente trabalho podem ter subestimado os teores ideais para o nutriente na palma-de-óleo.

\section{Conclusões}

1. Os métodos da chance matemática (ChM), DRIS e CND, utilizados na geração de faixas de suficiência, assim como o método DNR, na obtenção do nível crítico, são adequados para a avaliação do estado nutricional da palma-de-óleo.

2. Para a maioria dos nutrientes, as faixas de referência estimadas a partir dos métodos ChM, DRIS e CND apresentam valores próximos aos relatados na literatura; no entanto, os diagnósticos realizados com 
esses dados são divergentes, sobretudo quanto aos nutrientes $\mathrm{K}, \mathrm{Ca}$ e $\mathrm{Mg}$.

3. Os nutrientes $\mathrm{N}, \mathrm{Ca}, \mathrm{S}, \mathrm{Mn}$ e Zn figuram entre os mais frequentemente deficientes nos talhões avaliados.

4. A aplicação de micronutrientes deve fazer parte do plano de manejo nutricional da palma-deóleo, bem como as práticas que possam aumentar a disponibilidade de $\mathrm{Ca}$, como a calagem ou a gessagem.

\section{Agradecimentos}

Ao Conselho Nacional de Desenvolvimento Científico e Tecnológico (CNPq), pelo apoio financeiro; à Coordenação de Aperfeiçoamento de Pessoal de Nível Superior (Capes), por concessão de bolsa; ao engenheiro agrônomo Antônio José de Abreu Pina, à sua equipe da empresa Marborges Agroindústria e ao produtor rural José Ribamar Braga Matias, da empresa Agropecuária Canindé, por cederem os dados da pesquisa e pelo apoio operacional na coleta das amostras.

\section{Referências}

ÁGREN, G.I. Stoichiometry and nutrition of plant growth in natural communities. Annual Review of Ecology, Evolution, and Systematics, v.39, p.153-170, 2008. DOI: 10.1146/annurev. ecolsys.39.110707.173515.

ALVES, S.A. de O.; AMARAL, W.A.N. do; HORBACH, M.A.; ANTIQUEIRA, L.M.O.R; BRAGA, L.P.P.; DIAS, I.F. da S. A dendeicultura no Estado do Pará: cenário atual, entraves e perspectivas. Bioenergia em Revista: Diálogos, v.3, p.18-28, 2013.

BEAUFILS, E.R. Diagnosis and recommendation integrated system (DRIS). Pietermaritzburg: University of Natal, 1973. 132p. (Soil Science Bulletin, 1).

BEHERA, S.K.; RAO, B.N.; SURESH, K.; MANOJA, K. Soil nutrient status and leaf nutrient norms in oil palm (Elaeis guineensis Jacq,) plantations grown on Southern Plateau of India. Proceedings of the National Academy of Sciences, India Section B: Biological Sciences, v.86, p.691-697, 2016. DOI: 10.1007/s40011-015-0508-y.

CAMACHO, M.A.; SILVEIRA, M.V. da; CAMARGO, R.A.; NATALE, W. Faixas normais de nutrientes pelos métodos ChM, DRIS e CND e nível crítico pelo método de distribuição normal reduzida para laranjeira-pera. Revista Brasileira de Ciência do Solo, v.36, p.193-200, 2012. DOI: 10.1590/S010006832012000100020.

CARVALHO, A.J.C. de; FONTES, P.S.F.; FREITAS, M.S.M.; MONNERAT, P.H.; FONTES, A.G. Yellow passion fruit plant nutritional diagnosis at different phenological stages by the diagnosis and recommendation integrated system method.
Journal of Plant Nutrition, v.34, p.614-626, 2011. DOI: 10.1080/01904167.2011.538558.

CHEPOTE, R.E.; VALLE, R.R.; SANTANA, C.J.L. de. Resposta do dendezeiro à adubação mineral. Revista Brasileira de Ciência do Solo, v.12, p.257-262, 1988.

DIAS, J.R.M.; TUCCI, C.A.F.; WADT, P.G.S.; PARTELLI, F.L.; PEREZ, D.V.; ESPINDULA, M.C.; TOMIO, D.B. Antecipação do período de diagnose foliar em laranjeira 'Pêra' no Amazonas. Pesquisa Agropecuária Brasileira, v.48, p.757-764, 2013a. DOI: 10.1590/S0100-204X2013000700008.

DIAS, J.R.M.; TUCCI, C.A.F.; WADT, P.G.S.; SILVA. A.M. da; SANTOS, J.Z.L. Níveis críticos e faixas de suficiência nutricional em laranjeira-pêra na Amazônia Central obtidas pelo método DRIS. Acta Amazonica, v.43, p.239-246, 2013 b. DOI: $10.1590 / \mathrm{S} 0044-59672013000300001$.

FAIRHURST, T.H.; HÄRDTER, R. (Ed.). Oil palm: management for large and sustainable yields. Singapure: PPI: PPIC: IPI, 2003. $384 \mathrm{p}$.

FARNEZI, M.M. de M.; SILVA, E. de B.; GUIMARÃES, P.T.G. Diagnose nutricional de cafeeiros da região do Alto Jequitinhonha (MG): normas DRIS e faixas críticas de nutrientes. Revista Brasileira de Ciência do Solo, v.33, p.969-978, 2009. DOI: 10.1590/s0100-06832009000400021.

HERNANDES, A.; SOUZA, H.A. de; AMORIM, D.A. de; NATALE, W.; LAVRES JUNIOR, J.; BOARETTO, A.E.; CAMACHO, M.A. DRIS norms for 'Pêra' orange. Communications in Soil Science and Plant Analysis, v.45, p.2853-2867, 2014. DOI: 10.1080/00103624.2014.956933.

KURIHARA, C.H.; VENEGAS, V.H.A.; NEVES, J.C.L.; NOVAIS, R.F. de; STAUT, L.A. Faixas de suficiência para teores foliares de nutrientes em algodão e em soja, definidas em função de índices DRIS. Revista Ceres, v.60, p.412-419, 2013. DOI: 10.1590/S0034-737X2013000300015.

MALAVOLTA, E.; VITTI, G.C.; OLIVEIRA, S.A. de. Avaliação do estado nutricional das plantas: princípios e aplicações. 2.ed. São Paulo: Potafos, 1997. 319p.

NACHTIGALL, G.R.; DECHEN, A.R. DRIS norms for evaluating the nutritional state of apple tree. Scientia Agricola, v.64, p.282287, 2007. DOI: 10.1590/S0103-90162007000300011.

PALMELIT: PLANTING MATERIAL SOLUTIONS FOR THE TROPICS. Literature. Disponível em: <http://www.palmelit. com/en/literature $>$. Acesso em: 20 dez. 2014.

PARENT, L.-É. Diagnosis of the nutrient compositional space of fruit crops. Revista Brasileira de Fruticultura, v.33, p.321-334, 2011. DOI: 10.1590/S0100-29452011000100041.

RODRIGUES, M. do R.L. Resposta do dendezeiro (Elaeis guineensis Jacq.) à aplicação de fertilizantes nas condições do Médio Amazonas. 1993. 81p. Dissertação (Mestrado) Universidade de São Paulo, Piracicaba.

RODRIGUES, M. do R.L.; AMBLARD. P.; BARCELOS, E.; MACEDO, J.L.V. de; CUNHA, R.N.V. da; TAVARES, A.M. Avaliação do estado nutricional do dendezeiro: análise foliar (reformulada). Manaus: Embrapa Amazônia Ocidental, 2006. 9p. (Embrapa Amazônia Ocidental. Circular técnica, 26). 
SANTOS, E.F. dos; DONHA, R.M.A.; ARAÚJO, C.M.M. de; LAVRES JÚNIOR, J.; CAMACHO, M.A. Faixas normais de nutrientes em cana-de-açúcar pelos métodos ChM, DRIS e CND e nível crítico pela distribuição normal reduzida. Revista Brasileira de Ciência do Solo, v.37, p.1651-1658, 2013. DOI: 10.1590/S0100-06832013000600021.

SERRA, A.P.; MARCHETTI, M.E.; VITORINO, A.C.T.; NOVELINO, J.O.; CAMACHO, M.A. Determinação de faixas normais de nutrientes no algodoeiro pelos métodos CHM, CND e DRIS. Revista Brasileira de Ciência do Solo, v.34, p.105-113, 2010. DOI: 10.1590/S0100-06832010000100011.

TEIXEIRA, L.A.J.; TECCHIO, M.A.; MOURA, M.F.; TERRA, M.M.; PIRES, E.J.P. Normas DRIS e níveis críticos de nutrientes para videira 'Niagara Rosada' cultivada na região de JundiaíSP. Revista Brasileira de Fruticultura, v.37, p.247-255, 2015. DOI: 10.1590/0100-2945-409/13.

TOMIO, D.B.; UTUMI, M.M.; PEREZ, D.V.; DIAS, J.R.M.; WADT, P.G.S. Antecipação da diagnose foliar em arroz de sequeiro. Pesquisa Agropecuária Brasileira, v.50, p.250-258, 2015. DOI: 10.1590/S0100-204X2015000300009.

TRASPADINI, E.I.F.; WADT, P.G.S.; DIAS, J.R.M.; SCHMIDT, R.; PEREZ, D.V. Aplicação da distribuição normal reduzida na definição de nível crítico. Porto Velho: SBCS, 2014. 47p.

UEXKÜLL, H.R. von; FAIRHURST, T.H. The oil palm: fertilizing for high yield and quality. Bern: IPI, 1991. 79p. (IPI. Bulletin, 12).
URANO, E.O.M.; KURIHARA, C.H.; MAEDA, S.; VITORINO, A.C.T.; GONÇALVES, M.C.; MARCHETTI, M.E. Determinação de teores ótimos de nutrientes em soja pelos métodos chance matemática, sistema integrado de diagnose e recomendação e diagnose da composição nutricional. Revista Brasileira de Ciência do Solo, v.31, p.63-72, 2007. DOI: 10.1590/S010006832007000100007.

VIÉGAS, I. de J.M.; MÜLlER, A.A. (Ed.). A cultura do dendezeiro na Amazônia brasileira. Belém: Embrapa Amazônia Oriental; Manaus: Embrapa Amazônia Ocidental, 2000. 374p.

WADT, P.G.S.; ANGHINONI, I.; GUINDANI, R.H.P.; LIMA, A.S.T. de; PUGA, A.P.; SILVA, G.S. da; PRADO, R. de M. Padrões nutricionais para lavouras arrozeiras irrigadas por inundação pelos métodos da CND e chance matemática. Revista Brasileira de Ciência do Solo, v.37, p.145-156, 2013. DOI: 10.1590/S010006832013000100015.

WADT, P.G.S.; DIAS, J.R.M. Premissas para a aplicação do DRIS em espécies florestais e palmeiras. In: PRADO, R. de M.; WADT, P.G.S. (Ed.). Nutrição e adubação de espécies florestais e palmeiras. Jaboticabal: FCAV/CAPES, 2014. v.1, p.277-298.

WADT, P.G.S.; NOVAIS, R.F.; ALVAREZ V., V.H.; FONSECA, S.; BARROS, N.F. Valores de referência para macronutrientes em eucalipto obtidos pelos métodos DRIS e chance matemática. Revista Brasileira de Ciência do Solo, v.22, p.685-692, 1998. DOI: $10.1590 /$ S0100-06831998000400014.

Recebido em 1 de setembro de 2015 e aprovado em 10 de março de 2016 